\title{
Musi/ha/cking
}

\section{Ce que la musique fait au hacking (et inversement)}

Par Nicolas Nova (HEAD, Dijon) et François Ribac (Université de Dijon)

In Volume! La revue des musiques populaires, Editions Mélanie Seteun 2019, Musique et Hacking, 16 (2), pp.115-126.

\section{Résumé}

Dans cet article nous nous intéressons aux convergences entre hacking et pratiques musicales (musicking). Pour cela, nous mobilisons des terrains et époques variés ainsi que la sociologie et l'histoire des sciences. L'analyse de ces convergences nous amène d'abord à proposer une définition plus ouverte du hacking, où les amateurs ont toute leur place. Puis, nous montrons comment le hacking -au sens de la modification d'un système technique par une communauté d'usagers- peut nous aider à étudier la musique in situ et en action.

In this article we are interested in the convergences between hacking and musical practices (musicking). We mobilize various fields and periods as well as sociology and the history of science (STS). This analysis leads us, firstly, to propose a more open definition of hacking, where amateurs have a broadly role. Secondly, we show how hacking -the modification of a technical system by a community of users - can help us to study music in action.

\section{Mots clés}

Hacking, musiques populaires, musicking, études de sciences, innovations sociales Hacking, popular music, musicking, STS, social innovations

\section{Styles musicaux}

8 bit reggae, low fi, noise, musique improvisée, musique contemporaine, crooning

\section{Biographies}

Nicolas Nova, enseignant et chercheur à la Haute Ecole d'Art et de Design, Genève.

François Ribac, compositeur et enseignant chercheur à l'Université de Dijon, membre du laboratoire CIMEOS.

\section{Le hacking et ses pratiques}

Le terme de hack ou de "hacker culture" fait référence aux pratiques d'ingénierie se déroulant en dehors de la science ou l'industrie. Celles-ci se traduisent à la fois par différentes formes de bidouillage d'objets techniques - ce qui peut impliquer tout autant la programmation que l'électronique - et le partage de connaissances ou de ressources matérielles par des communautés de hackers.

Une première définition de ce terme correspond au fait de réaliser des hacks, c'est-à-dire de tirer parti de moyens techniques limités pour programmer, et plus largement créer, des objets et/ou des usages nouveaux. Historiquement, le terme apparaît à la fin des années cinquante autour d'un groupe de passionnés appartenant au club de modélisme ferroviaire du MIT ${ }^{\mathrm{i}}$. Contrairement à certains de leurs collègues du TMRC ${ }^{\mathrm{ii}}$, ces premiers hackers s'intéressaient moins à la conception soignée de 
répliques de trains, qu'à l'édification du réseau électrique et de communication permettant de faire rouler les maquettes de véhicule. C'est en effet le bricolage de ces systèmes électroniques qui a nécessité la mise en place de hacks, c'est-à-dire de solutions efficaces, élégantes et innovantes pour faire fonctionner l'ensemble, à partir d'une compréhension fine des moyens techniques à disposition (Levy, 1984). En transposant une telle démarche du train miniature à l'informatique, les hackers de l'époque se sont ensuite amusés à programmer un ancêtre du jeu de Pong sur l'énorme et intimidante machine à calculer IBM 704 (Levy, 2014, p. 15), puis, entre autres explorations, ont développé le jeu vidéo SPACEWAR sur l'ordinateur PDP-1.

C'est cette dimension du contournement, voire de l'exploitation de failles qui explique une autre connotation apparue ensuite du terme de hacking, couramment employée dans le domaine de la sécurité informatique. Cette seconde acception renvoie alors à la recherche intentionnelle de déverrouillage des protections logicielles et matérielles, en particulier dans le champ des télécommunications et de l'informatique. Un exemple couramment cité à cet égard est celui du hacker John Draper, dit " Captain Crunch », qui parvint en 1969 à passer des appels longue distance gratuitement en utilisant un sifflet possédant la même tonalité que le réseau téléphonique américain (Levy, 1984, p. 199). Si l'objectif est différent, il s'agit plutôt de forcer un système que de s'en inspirer, dans cette deuxième acception, la logique est similaire dans les deux cas puisqu'elle repose sur un intérêt profond à saisir le fonctionnement des objets techniques, et à l'exploiter afin de tester des usages nouveaux. Outre cette dimension de bidouillage créatif, la culture hacker correspond plus largement à un état d'esprit. Dans son enquête pionnière de 1984, le journaliste Steven Levy soulignait l'importance attachée à la liberté de l'information, la méfiance envers l'autorité, et surtout au jugement méritocratique ancré dans une évaluation des pratiques - c'est-à-dire dans la réalisation des hacks eux-mêmes, et non dans des critères d'âge, d'origine sociale ou de diplôme. Poursuivant cette analyse dans le champ du travail, le philosophe finlandais Pekka Himanen (2001) opposait même "l'éthique hacker" à celle du capitalisme héritée du protestantisme et décrite par Max Weber (1904/2010) : l'engagement du hacker dans une activité repose sur un intérêt intrinsèque pour celleci, et non pour le fait d'en retirer une rétribution pécuniaire.

Comme la montré Fred Turner (2008), des hackers - dans la première acceptation exposée plus haut -, certaines figures de la contre-culture nord-américaine des sixties, des ingénieurs, académiques et des acteurs institutionnels et industriels ont contribué à la conception et à la mise en œuvre de l'Internet, des médias numériques et à l'essor de la Silicon Valley. Cette rencontre, relayée par des ouvrages, des revues, des conférences, a très largement contribué à rendre l'informatique user-friendly et à imposer l'idée que des communautés pouvaient naître grâce à des réseaux de télécommunications et l'usage de PC (Personal Computer). Ce point est d'importance, le hacking ne renvoie pas uniquement au fait de modifier des systèmes techniques mais aussi au fait que ces pratiques prennent place dans des communautés de savoirs, d'échanges, de compétitions, communautés qui se retrouvent sur la toile, au grand jour ou dans les confins du Darknet (Stamboliyska 2017).

De nos jours, cette double composante - bidouillage et communauté organisée de hackers se retrouve dans les deux pôles du hacking les plus couramment perçus ; un hacking opposé aux pouvoirs et aux multinationales qui pénètre les systèmes pour mettre à jour des abus, par exemple celui des Anonymous et un hacking cynique et menaçant, qui fracture des sites et des ordinateurs pour rançonner particuliers et entreprises. Entre ces deux polarités, toutes sortes de communautés forcent ou réaménagent des systèmes techniques, produisent des effets non prévus dans les scripts originaux 
des concepteurs et contribuent à de nouveaux usages. On l'aura compris, si le terme de hack, et la culture hacker, ont pendant longtemps fait référence exclusivement à la culture informatique, celle-ci a prospéré dans d'autres domaines. En premier lieu dans le champ des réseaux, comme on vient de l'évoquer, mais aussi dans toutes sortes d'activités et de sphères sociales : la vie de tous les jours ("life hack"), le bricolage et le DIY individuels ou collectifs iii, le monde professionnel ("corporate hacking") et bien entendu la musique, comme nous le verrons plus loin.

\section{Les lumières des STS et de David Edgerton}

Tel qu'il est couramment défini, le hacking consiste, d'une part, à forcer et/ou détourner des systèmes techniques et, d'autre part, à ce que ces pratiques fassent émerger des communautés de hackers et dans certains cas d'usagers. Ce mouvement par lequel le monde social s'approprie des savoirs et des objets issus de l'industrie et/ou de la science a déjà été bien documenté dans les STS $^{\text {iv }}$ dans des situations et des époques variées par les historiens des sciences (par exemple Jacob et Stewart 2004) ${ }^{\mathrm{v}}$ ou la sociologie des usages des télécommunications (Jaureguiberry et Proulx, 2011). De même, la sociologie de la traduction, que l'on appelle aussi la sociologie des réseaux, a abondamment documenté la différence souvent patente entre le script prévu par les concepteurs d'une technologie et ses usages effectifs lorsque celle-ci trouve un espace dans le monde social (Akrich 1987 et Akrich, Callon \& Latour 2006). Sans qu'il soit question de présenter ce vaste corpus comme un tout homogène, une de ses constantes est de montrer que loin d'être dotée de propriétés propres toute technologie donne lieu à des controverses, des transformations, des déclinaisons, des usages imprévus, en bref que son destin et ses usages dépendent au moins autant de sa structure matérielle et des compétences et des objectifs de ses promoteurs que de ce que ses usagers en font (ou pas). Pour résumer ce premier point, une technologie n'existe pas en soi, elle ne prend sens que lorsqu'elle s'inscrit dans le monde social et des usages et, ce point est ici crucial, elle est presque toujours détournée, transformée, récupérée, appropriée. Mieux, ses usages imprévus sont souvent une dimension consubstantielle de la diffusion des objets techniques. De ce point de vue, le hacking rend visible et décline à l'âge électronique puis informatique une composante essentielle, quasi ontologique, et antérieure aux sixties, de toute société.

Pour comprendre la fluidité des technologies, l'infinie variété de leurs déclinaisons et de leurs usages, les travaux de l'historien David Edgerton méritent également d'être mobilisés. S'appuyant sur une abondance de terrains, de pays et d'époques, Edgerton (2011) critique la façon dont l'histoire des technologies décrit souvent un enchaînement, uniforme et irréversible, de révolutions technologiques liées à des énergies ; charbon, machine à vapeur, électricité, pétrole, numérique etc. À contrario, il montre comment les usages d'une même technologie varient non seulement dans le temps mais aussi selon les pays et les situations sociales. Ainsi, dans la région Suame Magazine au Ghana, une des zones les plus industrialisées d'Afrique, des biens (machines outils, voitures, électroménager etc.) considérés comme obsolètes dans le monde occidental sont réparés, entretenus et fonctionnent parfaitement durant des décennies. Les mécaniciens ghanéens forgent des savoir-faire techniques qui leur permettent de contourner les chaînes de compétences (mode d'emploi, ingénieurs etc.) dont ils ne peuvent pas disposer pour des raisons économiques. En d'autres termes, les mécaniciens ghanéens hackent des techniques et des objets.

Outre cet éclairage décentré du seul monde occidental (là-dessus voir Chakrabarty 2000), Edgerton s'inspire de l'écrivain Patrick Chamoiseau (1992) pour parler de technologies créoles. Ce 
terme désigne pour lui des systèmes ou objets qui, transplantés des pays riches vers les pauvres, trouvent d'autres fonctionnalités. Et de montrer ainsi comment les bicyclettes, instruments sportifs et de loisirs à l'origine (Oudshoorn \& Pinch 2003), devinrent des moyens de transports majeurs en Asie dans les années 1930-1950 puis comment les "bicyclettes asiatiques" se sont à nouveaux hybridées pour donner naissance au pousse-pousse. De plus, cette importance de ce que l'on pourrait appeler la circulation horizontale des savoirs et des objets est également vraie pour Edgerton dans un même espace. Par une série d'exemples allant de la composition de l'armée allemande lors de la seconde guerre mondiale (où les chevaux étaient bien plus nombreux que les chars et furent tout autant décisifs lors des combats) au parc automobile à Chicago dans les années 1920 (qui comptait nombre de voitures électriques), l'historien anglais montre que de nombreuses technologies voisinent alors que l'on insiste habituellement sur une seule technologie (ou énergie) et à qui l'on attribue un rôle moteur. L'hybridation de la bicyclette, et plus largement cette conception de technologies créoles, peuvent être comprises comme des formes de hack au même titre que les détournements d'objets techniques ou les contournements de systèmes informatiques.

Dernier point capital chez Edgerton, pour qu'une innovation technologique trouve son public et se pérennise, il est absolument nécessaire que sa part de nouveauté soit réduite. Autrement dit et à rebours d'une conception où les révolutions technologiques changent radicalement la donne, les transformations notables de pratiques et l'apparition de "nouveaux objets" s'appuient beaucoup plus sur des déplacements voire même des changements à la marge que sur des bouleversements. Si ce dernier point a bien été appréhendé par les STS, qui ont souvent mis l'accent sur les continuités dans les innovations (par exemple Pinch \& Trocco 2002 sur le moog), Edgerton nous montre que ces continuités « fonctionnent » également dans un espace et une temporalité identiques.

Qu'est-ce que les STS et la contribution d'Edgerton, encore trop méconnue dans le monde francophone, nous apprennent sur le hacking ? Premièrement, et nous l'avons déjà dit plus haut, ces travaux permettent de situer le hacking dans une généalogie de pratiques, bien antérieures aux années soixante. Deuxièmement, les nombreux terrains mobilisés par Edgerton nous apprennent que le détournement, le contournement, les nouveaux usages s'appliquent à de nombreux objets, types de systèmes et qu'ils sont déclinés dans des territoires et configurations sociales très différentes et, ce point est fondamental, pour des raisons différentes que celles qui mobilisent les hackers dédiés au bien commun ou hostiles. À partir de ces points, il nous semble que la compréhension du hacking est plus nuancée, plus panoramique aussi et qu'elle s'appuie sur une approche de la technologie également plus équilibrée.

\section{Ce que la musique fait au hacking}

Quid du hacking musical ? Intéressons nous d'abord à des styles musicaux où les façons de faire semblent similaires aux composantes "classiques" du hacking qui ont été proposées plus haut ; le fait qu'une communauté d'usagers force des systèmes et/ou détourne des objets et partage ses découvertes. Le circuit bending (figure 1) qui consiste à faire circuler du courant de façon imprévue dans des circuits intégrés de machines (par exemple des jouets) afin de faire surgir de nouvelles sonorités trouve aisément sa place dans cette première catégorie. 


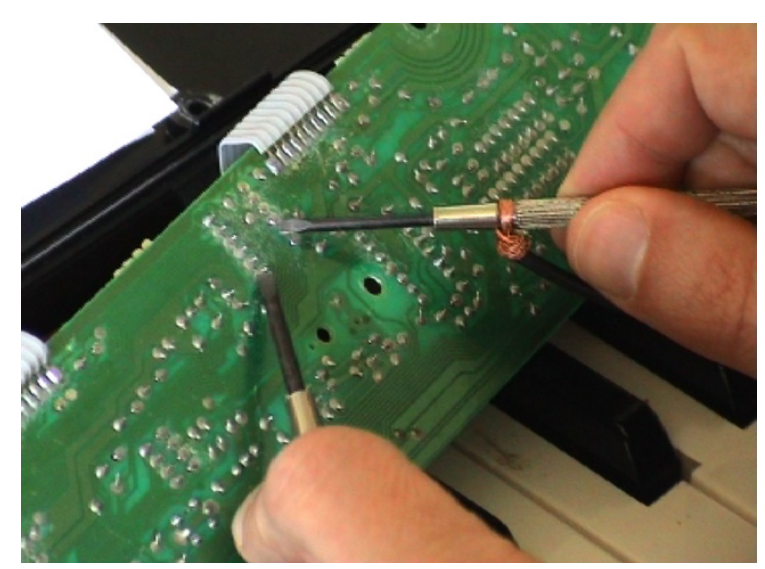

Figure 1 : Circuit bending : deux tournevis reliés par un fil électrique permettent de faire circuler du courant de façon aléatoire dans les circuits intégrés et de découvrir des sons inédits. Photographie François Ribac.

On pourrait également mentionner la musique noise où fréquemment les usagers détournent et assemblent toutes sortes de générateurs de sons (Cf. le texte de Sarah Benhaim dans ce dossier), la vaporwave (où l'on sample et ralentit des tubes de pop) ou encore la low fi, cette forme de rock (ou de pop) qui privilégie des formes peu coûteuses et souvent domestiques d'enregistrement et de diffusion de sa musique. On pourrait également évoquer certaines composantes de la musique improvisée où les musicien-n-e-s bricolent leurs instruments avec des objets, choses, détritus initialement non destinés aux usages qu'ils/elles en font ou encore les « usages limites » d'instruments de musique que l'on trouve dans la musique de compositeurs contemporains tels que Helmut Lachenmann ou Giacinto Scelsi.

On remarquera que cette liste fait voisiner des styles et des pratiques qui, au-delà de leurs formes de production et des réseaux au sein desquels ils prennent place, ont en commun de faire du détournement des objets un acte volontaire, "radical" diraient même certain-e-s protagonistes de ces mondes. Conséquemment, les protagonistes de ces mondes insistent sur la dimension éthique de leurs pratiques, leur indifférence à la commercialisation et le désir de ne pas être manipulé etc. Le point commun avec certaines formes progressistes de hacking est patent.

\section{a) Le reggae 8-bit, une technique créole}

Justement, le cas du reggae 8-bit - un micro-genre musical qui consiste à produire et jouer du reggaedub avec des consoles de jeu vidéo ou des ordinateurs munis de processeurs 8-bit - illustre ce lien entre musique, pratiques hackers, et la notion de techniques créoles proposée par David Edgerton. L'un d'entre nous a ainsi décrit ailleurs ces pratiques par le biais d'une enquête de terrain en Europe, montrant comment des consoles de jeu vidéo japonaises et des ordinateurs nord-américains avaient été détournés à cet effet (Nova, 2014 ; Nova, 2017). Pratiquée tant par des musiciens de la scène dite "chiptune", qui emploient des machines "low-tech" telles que la Game Boy (Nintendo), l'Amiga (Commodore), ou le C64 (Commodore) disponibles dans les années 1980, que par des producteurs de musique électronique à l'affut de sonorités et de terrains d'expérimentations nouveaux, l'appellation "reggae 8-bit" renvoie en fait à des pratiques musicales multiples. Une majorité d'artistes se limite à prélever des échantillons sonores ou à utiliser ces ordinateurs et consoles momentanément dans leurs compositions ; par exemple pour affubler des riddims reggae ${ }^{\mathrm{vi}}$ classiques de samples tout aussi connus dans la culture vidéoludique. D'autres poussent leur passion plus loin et créent leurs propres instruments à partir de ces machines des années 1980. C'est chez ceux-ci que l'on peut trouver des pratiques proches du hacking et qui débouchent sur une technique créole. Prenons ici l'exemple de la MIDIbox SID synthesizer "conçue" par les fondateurs de Jahtari (voir 
Nova 2014 pour plus de détails). À côté de ses activités de production, ce label allemand propose aux musiciens intéressés un synthétiseur spécialement dédié au reggae 8-bit. Il s'agit d'un appareil sommaire, monté à la demande exclusivement pour les amis et les proches et dont ils ont fixé le prix de vente à 1150 euros. Celui-ci est formé de deux blocs de synthèse sonore, munis d'une interface de contrôle (boutons, potentiomètres, indicateurs visuels) insérés dans une coque d'origine de Commodore C64. Chacun des blocs synthétiseurs comprend quant à lui le microprocesseur sonore d'un ancien C64, le "SID” (Sound Interface Device) qui permet de générer les sons si particuliers et identifiables du jeu vidéo des années 1980. Or, ni cet ordinateur, ni ce composant ne sont encore fabriqués actuellement - malgré l'existence de copies de mauvaise qualité - ce qui implique donc de surveiller les plateformes de vente en ligne d'objets de seconde-main, d'acheter régulièrement des C64 pour en extraire les microprocesseurs sonores, et récupérer les coques en plastique. Lesquels éléments sont réutilisés ensuite, hybridés avec des composants plus récents pour produire la MIDIbox SID synthétiseur.

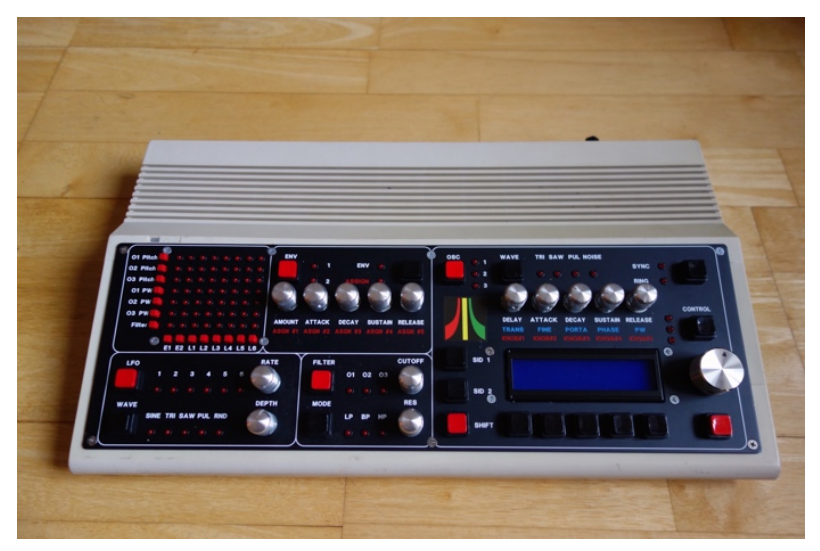

Figure 2 : MIDIbox SID synthesizer (Jahtari, 2014)

La création de cet objet hybride - c'est à dire les hacks nécessaires à sa production - rappelle la notion de technique créole proposée par Edgerton : "la diffusion de techniques singulières souvent dérivées de 'vieilles techniques"' et renvoyant à des "dérivés locaux de quelque chose originaire d'ailleurs" (Edgerton, 2011, p. 120). Comme décrit ailleurs (Nova, 2017), le MIDIbox SID peut être décrit comme une « dérivée de vieilles techniques » avec ses processeurs sonores SID « low tech » et leur sonorités identifiables qui renvoient à une culture bien spécifique. De même, la transposition spatiale et temporelle soulignée par Edgerton est aussi présente. Les bricoleurs de Jahtari étant des "Allemands de l'Est", comme ils se plaisent à le rappeler, qui modifient et combinent des technologies nord-américaines low-tech (les SID, le C64) et sud-asiatiques (fournisseurs de composants électroniques high-tech actuels) pour faire évoluer un genre musical caribéen lui-même hybride.

\section{b) Bifurcations sans savoirs techniques : l'exemple du microphone}

La deuxième façon d'envisager le hacking dans les mondes musicaux concerne des processus au cours desquels des usages, des outils, des instruments et des systèmes en viennent à être fracturés et/ou décalés, mais sans que le registre du détournement soit forcément mis en avant par les acteurs. À bien y regarder/écouter, bien des objets et pratiques aujourd'hui naturalisées sont pourtant bien le résultat d'une combinaison de hacks.

Considérons par exemple l'usage en scène des microphones. Développés dans le cadre des recherches sur l'électrification du signal menées par les firmes téléphoniques dans les années 1920 
(Gelatt 1977, Millard 1995, Adams \& Butler 1999, Taylor, Katz \& Grajeda 2012), les micros étaient originellement utilisés pour recueillir le signal dans les studios d'enregistrement et dans les radios, en particulier aux USA. Si le son recueilli était bien amplifié électriquement pour être gravé sur un support lors des séances d'enregistrement ou "broadcasté » lors des émissions de radio, ni les ingénieurs en télécommunications, ni les opérateurs des studios n'avaient destiné les microphones à un usage scénique ; amplifier signifiait augmenter électriquement le niveau du signal au sein du réseau de circulation du son pour mieux le capter 'tel quel", pas augmenter son volume afin qu'il soit diffusé plus fort lors de performances publiques. Ainsi, lorsque des interprètes se produisaient devant un public lors d'une émission de radio, une situation que Hollywood a documenté dans de nombreux films ${ }^{\text {vii }}$ les voix ou les instruments étaient «seulement » captés par un micro pour l'envoyer "dans les tuyaux" mais pas amplifiés dans une sonorisation ad hoc à destination du public. En somme, les ingénieurs électriques amplifiaient le signal et non pas le son ou la musique. À la même époque, certains vocalistes comme Rudy Vallée ou Bing Crosby utilisaient néanmoins des mégaphones pour mieux se faire entendre en concert tandis que les partis politiques commençaient à utiliser des amplificateurs, des haut-parleurs et des microphones pour leurs meetings (Devine 2013).

Ces mêmes chanteurs/euses, que l'on appellerait bientôt des crooners, eurent alors l'idée de coupler les microphones des studios de radio et d'enregistrement avec des amplificateurs (par exemple celui d'une radio domestique) et de les utiliser en scène (Lockheart 2003). Ce qui est ici important de signaler est que l'amplification (au sens où l'on parle aujourd'hui de musique amplifiée) servit tout autant à augmenter le niveau sonore des voix et à les distinguer des orchestres, qu'elle permit aux crooners de moduler leur voix et de chanter des pianissimo et des nuances faibles même lorsque l'orchestre jouait fort. Comme l'a montré McCracken (2015) avec l'exemple de Rudy Vallée, l'un des premiers crooners, immense star (oubliée) de la radio et pionnier du microphone, son usage du microphone donna lieu à de violentes polémiques sur les capacités vocales de ceux et celles qui y recourait, accusations allant souvent de pair avec des accusations d'homosexualité à l'encontre des hommes. Comme le rappelle le titre de l'ouvrage de McCracken ; "Real men don't sing" et certainement pas avec un microphone. Bruce Johnson (2000) a d'ailleurs montré que, dans le monde du jazz, c'est surtout les femmes qui adoptèrent le nouvel objet souvent dédaigné par les hommes.

Dès lors, peut-on considérer l'usage du microphone comme une sorte de hacking ? Cela y ressemble à maints égards. Il y a bien une communauté d'usagers qui opère le déplacement, un objet et un réseau technique existants (l'amplification du son) affectés à un usage imprévu et, peut-être le plus important, la naissance de nouvelles configurations techniques et spatiales, de nouvelles compétences et des métiers inédits (les sonorisateurs), de nouvelles expériences d'écoute, de nouveaux mondes musicaux (les crooners), de nouveaux objets qui recomposent la physionomie de la « musique ». Comme le montre Edgerton dans d'autres sphères sociales, les micros passent du studio à la scène par une sorte de glissement d'un lieu (les studios d'enregistrement et de radio) à un autre (la scène) en s'appuyant sur des pratiques et des objets déjà existants. Et surtout, les crooners/euses font surgir des ressources inconnues d'un instrument, détournent des objets de leur usage habituel sans avoir la compréhension d'un ingénieur, sans comprendre explicitement comment un système technique fonctionne. De plus, ces détournements ne concernent pas seulement le fait d'utiliser un micro en scène mais s'expriment par la naissance d'une nouvelle façon de chanter, par le développement et la diversification d'un style qui se déploie tout au long des années 1960, 1940 et 1950 sur les scènes comme dans les studios (Granata 1999) et qui influencera les styles suivants et en particulier le rock' n' roll.

D'autres exemples, plus récents, comme l'utilisation des platine disques et des répertoires enregistrés et dans le rap ou les usages des cassettes audio sont très largement similaires à ceux du micro des crooners. Ils montrent en outre que nombre de ces détournements émanent d'amateurs (pour plus de détails voir Ribac 2005). 


\section{c) Apprentissages}

Notre troisième entrée concerne une fois encore les musiques populaires mais se décline dans les processus d'apprentissage de ces musiques et implique des amateurs.

Plusieurs études, réalisées avant, pendant et après la dissémination des outils numériques et du Web (Bennett 1980, Green 2001, Ribac 2005, 2007, 2010 et 2012) ont en effet montré que le processus d'apprentissage du rock, du hip hop et de la techno se déroulent non seulement dans des cadres collectifs (par exemple les groupes de rock), mais que cette phase est précédée d'un usage intensif et solitaire des outils de reproduction sonore et des supports enregistrés. Bennet a ainsi montré, un point qui a beaucoup frappé Howard Becker, que des adolescents vivant dans les montagnes du Colorado à la fin des années 1970 étaient capables de reproduire des solos de guitare de Frank Zappa sans jamais avoir pris un cours de guitare ni joué dans un groupe. Ce recours à des instructeurs non humains, les supports enregistrés et leurs lecteurs, est un fait central dans l'apprentissage des musiques populaires et ce depuis que les phonographes et la radio ont fait leur entrée dans l'espace domestique. Il est déjà documenté dans la biographie d'un Bing Crosby apprenant la musique avec la radio et chantant avec le gramophone familial dans les années 1920 (Giddins 2001, Martin et Crosby 2003) ou encore dans les récits des rockers des sixties comme McCartney ou Keith Richard (2010) engagés dans leurs groupes respectifs parce qu'ils jouaient à la perfection des morceaux de rock'n 'roll appris avec des disques et des tourne-disques Dansette. Des ethnographies réalisées au milieu des années 2000 montrent des adolescent-e-s en phase d'apprentissage bidouillant des systèmes multipistes avec des magnétophones à cassettes à la maison, utilisant la souris d'un ordinateur pour générer des sons à la place d'un clavier, faisant circuler de la modulation dans des configurations assez improbables (Ribac 2007, 2010 et 2012). Dans un monde où les mashups viii , la vaporwave et même les façons d'écrire des SMS (Serres 2012) ont également été initié par des amateurs, ces innovations s'inscrivent là aussi dans une généalogie de hacks réalisés par des (groupes de) personnes sans compétences reconnues.

\section{Conclusion(s)}

\section{a) Ce que le hacking fait à la musique}

Dans un ouvrage qui a fait date dans les études musicales, Christopher Small (2011) a proposé le terme de musicking pour décrire la multiplicité des significations, des pratiques, des usages et des collectifs qui composent ce que l'on appelle communément "la musique“. Difficile à traduire en français, le suffixe "-ing" signifie le "en train de se faire" ou, pour le dire autrement, que la musique vient à nous dans sa mise en œuvre et ses usages. Ce qui implique de penser, d'appréhender, et d'étudier la musique en action comme dirait Tia DeNora (2011). De ce point de vue, le hacking, tel que nous l'avons défini au début de ce texte, permet de d'appréhender les pratiques musicales en portant attention autant aux gestes et aux objets (ce que l'on appellerait la technique) qu'aux formes de sociabilités. C'est à la conjonction entre ces deux pôles que l'on peut alors observer, écouter et repérer des innovations, des ruptures mais aussi des continuités stylistiques et matérielles, par exemple le dub et les processeurs des appareils des années 1950 dans un nouveau style : le 8 bit reggae. Appréhender ainsi le musicking permet à notre sens de mieux comprendre comment de nouvelles communautés musicales surgissent et les divers registres qui donnent corps à ces styles de vie qui émergent et ce sans l'aveuglement des approches analytiques ou déterministes ni la distance du sociologisme qui rabat pratiques et objets à des reflets (Hennion 1993). 


\section{b) Ce que les STS et la musique font au hacking}

Réciproquement les études de science et en particulier le travail d'Edgerton nous rappellent que pour s'imposer et perdurer toute technologie est nécessairement hackée. Autrement dit, les différentes formes de hacking nées à l'âge électronique et informatique doivent, premièrement, être replacées dans des généalogies historiques et, deuxièmement, considérées comme une de formes par lequel un segment du monde social s'approprie des objets, des dispositifs, des technologies etc. Si, comme on l'a vu avec l'exemple du 8 bit reggae, il existe des formes quasi ingénériales de bidouillage dans la musique, le microphone des crooners et l'apprentissage des musiques populaires montrent que les fractures, les bifurcations et les usages détournés peuvent advenir, d'une part, sans une volonté explicite de rupture et, d'autre part, sans qu'il soit nécessaire de comprendre et d'analyser le fonctionnement des systèmes. Les amateurs sont souvent à l'origine de hacks qui ont modifié systèmes techniques et organisations sociales. Autrement dit, non seulement il n'est pas nécessaire de savoir comment fonctionne un objet pour l'utiliser (et heureusement !) mais cette méconnaissance peut même aider à en faire autre chose. Enfin, le savoir qui accompagne un système technique ou une machine est fondamentalement social, c'est par le biais de la communauté qui l'utilise que j'apprendssouvent implicitement- à l'utiliser (Collins 2010). Le mode d'emploi est sûrement utile mais si le monde social ne me dit pas comment (et ce comment peut très fortement varier) l'utiliser, je n'y arriverai pas. Si les théories sur le hacking devraient se musiquer et la musique se hackiser cela donne donc musi[ha]cking.

\section{Bibliographie}

Adams S.B. et Butler O.R. (1999), Manufacturing the future, a History of Western Electric, Cambridge, Cambridge University Press.

Akrich M. (1987), "Comment décrire les objets techniques ?”, Techniques et culture, pp. 49-64.

Akrich, M., Callon, M. et Latour, B. (dir.) (2006), Sociologie de la traduction. Textes fondateurs, : Presses des Mines, Paris.

Bennett H.S. (1980), On becoming a rock musician, Amherst, MA, University of Massachussets Press.

Chakrabarty D. (2000), Provincializing Europe, Princeton University Press, Princeton.

Devine K. (2013), “A mysterious music in the air", Popular Music History, vol. 8, n¹, pp. 5-28.

Chamoiseau, P. (1992), Texaco, Paris, Gallimard.

Collins H. (2010), Tacit and explicit knowledge, University of Chicago Press, Chicago.

DeNora T. (2011), Music in Action. selected essays in sonic ecology, Ashgate, Farnham.

Edgerton D. (2011), Shock Of The Old: Technology and Global History since 1900, London, Profile Books.

Edgerton D. (2013), Quoi de neuf? Du rôle des techniques dans l'histoire, traduit par Jeanmougin C., Paris, Seuil.

Gelatt R. (1977), The fabulous phonograph 1877-1977 (second edition), London, Cassell.

Giddins G. (2001), Bing Crosby, a pocketful of Dreams: The Early Years, 1903-1940., Little, Brown and Company.

Granata C.L. (1999), Sessions with Sinatra: Frank Sinatra and the art of recording, Chicago, A Cappella Books.

Green L. (2001), How popular musicians learn, a way ahead for music education, Ashgate, Aldershot.

Hennion A. (1993), La Passion Musicale, une sociologie de la médiation, Paris, Métailé.

Himanen, P. (2001), L'éthique hacker, Paris : Exils. 
Jacob M.C. et Stewart L. (2004), Practical matter. Newton's science in the service of Industry \& Empire, 1687-1851, Cambridge, Harvard University Press.

Jaureguiberry, F., Proulx, S. (2011), Usages et enjeux des technologies de communication, Toulouse: Erès.

Johnson B. (2000), The inaudible music, jazz, gender and australian modernity, Currency Press. Levy S. (2010), Hackers, Sebastopol, O’Reilly books.

Lockheart P. (2003), “A history of Early Microphone Singing, 1925-1939: America Mainstream popular Singing at the Advent of electronic microphone Amplification", Popular Music and Society, 26:3, 367-385.

Martin P. et Crosby B. (2003), Call me lucky, Bing's Crosby's own story, Da Capo Press, Boston. McCracken A. (2015), Real men don't sing. Crooning in american culture, Durham, Duke University Press.

Millard A. (1995), America on record. A history of recorded sound, Cambridge, Cambridge University Press.

Nova N. (2014), 8-bit Reggae, collision and creolizatiion, Volumique.

Nova, N. (2017), "Démonter, extraire, combiner, remonter. Commodore 64 et créolisation technique", Techniques et Culture, $67: 1,116-133$.

Oudshoorn, N. et Pinch, T. (dir.) (2003), How Users Matter: The Co-Construction of Users and Technology (Inside Technology), Cambridge, The MIT Press.

Pinch T. et Trocco F. (2002), Analog Days. The Invention and Impact of the Moog Synthesizer, Cambridge, Harvard University Press.

Ribac F. (2005), "Sur l'importance des disques et du recording dans la musique populaire et la techno", Mouvements $n^{\circ}$ 42, Paris, novembre/décembre, pp. pp.70-81.

Ribac F. (2007), "La circulation et l'usage des supports enregistrés dans les musiques populaires en Ile de France", Commandé par le programme interministériel «Culture et Territoires en Ile de France », le Ministère de la Culture (Bureau des Écritures) et le Conseil général de Seine-SaintDenis.

Ribac F. (2010), "L'autre musique de chambre, comment de jeunes adolescent-es ont appris la musique", Actes du colloque international enfance et cultures.

Ribac F. (2012), “Quand l'amateur rend le numérique analogique ; l'exemple des musiques populaires", Revue Anthropologie des connaissances, Vol. 6., pp. pp.717-741.

Richard K. (2010), Life, Paris, Hachette/Littlehampton.

Serres M. (2012), Petite poucette, Paris, Le Pommier.

Small C. (2011), Musicking: The Meanings of Performing and Listening (Music Culture), Hanover, Wesleyan University Press.

Stamboliyska R. (2017), La face cachée de l'Internet, Paris, Larousse.

Taylor, T.D., Katz, M. et Grajeda, T. (dir.) (2012), Music sound and technology in America, Durham, Duke University Press.

Turner F. (2008), From Counterculture to Cyberculture: Stewart Brand, the Whole Earth Network, and the Rise of Digital Utopianism, University of Chicago Press.

Weber, M. (2010), L'éthique protestante et l'esprit du capitalisme, Paris, Pocket/Plon, collection Agora. 
i $\quad$ MIT = Massachusetts Institute of Technology, centre de recherche et université, connu pour ses nombreuses contributions aux innovations du $20^{\mathrm{e}}$ et $21^{\mathrm{e}}$ siècle.

ii TMRC signifie Tech Model Railroad Club et désigne ne association d'étudiants du MIT créé en 1946.

iii Un intérêt que l'on retrouve dans l'avènement de ces lieux de bricolage et d'apprentissage que sont les hackerspaces et autres fab labs. Relevons d'ailleurs que ces derniers sont aussi originaires du MIT, une université qui a toujours entretenu un rapport d'ouverture à ses cours, ses ateliers et ses machines. Promus par un chercheur du MIT Medialab, Neil Gershenfeld, les fab labs peuvent être lus comme une émanation récente de cette hacking culture.

iv L'acronyme STS (Science and Technology Studies) désigne un vaste corpus de travaux et de chercheur.e.s qui considèrent les sciences et les technologies comme des mondes sociaux.

v Jacob et Stewart (2004) ont documenté la déclinaison instrumentale des théories newtoniennes en Grande-Bretagne au XVIIIe siècle.

vi Le terme riddim, déformation de l'anglais rhythm («rythme») provient du patois jamaïcain; il désigne la structure reprise de morceau en morceau ("versions") dans le reggae.

vii Par exemple dans la série de films intitulé "The Big Broadcast of" débutée dans les années 1930 à Hollywood.

viii Un mashup consiste en la création d'une chanson, ou composition musicale, à partir de deux ou plusieurs autres chansons déjà existantes. 